\title{
Les réseaux des voies navigables français et européens
}

\section{French and european navigable waterway systems}

\author{
R. Tenaud \\ Chef du Service Technique Central \\ des Ports Maritimes et des Voies Navigables
}

Historique sommaire de la navigation fluviale

Le poids du passé est particulièrement important.

La voie d'eau a été utilisée par les premières civilisations et fut jusqu'au siècle dernier le seul moyen de transport de marchandises en grandes quantités.

On a d'abord emprunté les rivières (1) dans leur état naturel. Trois millénaires avant J-C., les Egyptiens naviguaient déjà sur le $\mathrm{Nil}$ avec des barques de près de $50 \mathrm{~m}$ de longueur.

En Gaule, beaucoup de rivières étaient parcourues par des barques à fond plat qui remontaient loin vers les sources. Selon Strabon, il y aurait même eu des portages de marchandises pour franchir les seuils entre deux bassins. Au Moyen-Age, la navigation sur la Seine et ses principaux affluents était très développée. Beaucoup de bateaux étaient très sommairement construits et ne pouvaient pas remonter le courant. A l'extrémité aval de leur parcours, on les détruisait pour récupérer le bois (c'était le "déchirage").

Les techniques d'aménagement des rivières apparurent très tôt. Dès le début de notre ère, les Chinois inventèrent une forme primitive de barrage mobile pour augmenter la hauteur d'eau dans les rivières, ainsi que le canal latéral pour contourner une section de cours d'eau.

Pour suppléer au manque de rivières, des canaux artificiels furent creusés, déjà par les Assyriens et les Romains. Ces canaux furent particulièrement développés à partir du XII ${ }^{\mathrm{e}}$ Siècle en Flandre où le terrain était très favorable et permettait de faire de grandes longueurs de canaux au même niveau.

Le franchissement par des bateaux d'une dénivellation entre deux plans d'eau devenait un problème crucial pour le développement de la navigation fluviale. Les premiers ouvrages construits dans ce but furent très proba-

(1) Le terme de "rivière" est consacré en matière de navigation fluviale pour tous les cours d'eau, même les fleuves. blement les "overdrach" sur l'Yser. Il s'agissait de plans inclinés en bois où l'on faisait glisser les bateaux à sec. Mais ce système était peu pratique.

L'écluse a été conçue au XIV ${ }^{\mathrm{e}}$ Siècle. On ne sait pas d'une façon certaine si Léonard de Vinci en est l'inventeur ou s'il a seulement apporté un perfectionnement décisif aux idées d'ingénieurs hollandais en mettant au point un système de portes simples et robustes, les portes busquées, qui sont encore très fréquemment utilisées de nos jours.

Dans tous les pays où la géographie était favorable, il en résulta un développement important du réseau des voies navigables.

La France ouvrit l'ère des grandes réalisations avec la construction du Canal de Briare et du Loing entre la Seine et la Loire. Commencé en 1604, terminé en 1646 , il fut le premier canal qui franchit un seuil entre deux bassins. Le célèbre Canal du Midi, chef-d'œuvre de Riquet, fut achevé en 1681. D'autres réalisations suivirent, en particulier le Canal du Centre (1791), le Canal de St-Quentin (1810), le Canal du Rhône au Rhin et le Canal de la Marne au Rhin (1834).

Les écluses avaient en général 5,20 m de largeur et de 30 à $38 \mathrm{~m}$ de longueur. Les hauteurs de chute (dénivellation entre deux biefs adjacents) étaient limitées à $3 \mathrm{~m}$ environ par les possibilités techniques de construction et les problèmes de l'alimentation en eau. Celle-ci était effectuée par gravité, à l'aide de "rigoles", à partir des cours d'eau voisins, ou de réservoirs d'accumulation (Réservoir de St-Ferréol sur le Canal du Midi). De ce fait, ces canaux comportaient un nombre considérable d'écluses: 189 par exemple sur le Canal de Bourgogne, long de $242 \mathrm{~km}$.

Ces canaux présentaient un "mouillage" (ou profondeur d'eau) de $1,60 \mathrm{~m}$ à $2,00 \mathrm{~m}$, permettant le passage de bateaux portant de 180 à $300 \mathrm{t}$ de marchandises.

$\mathrm{Au}$ milieu du XIX ${ }^{\mathrm{e}}$ Siècle, tous les voies actuelles étaient tracées, à une exception près (le Canal du Nord). 


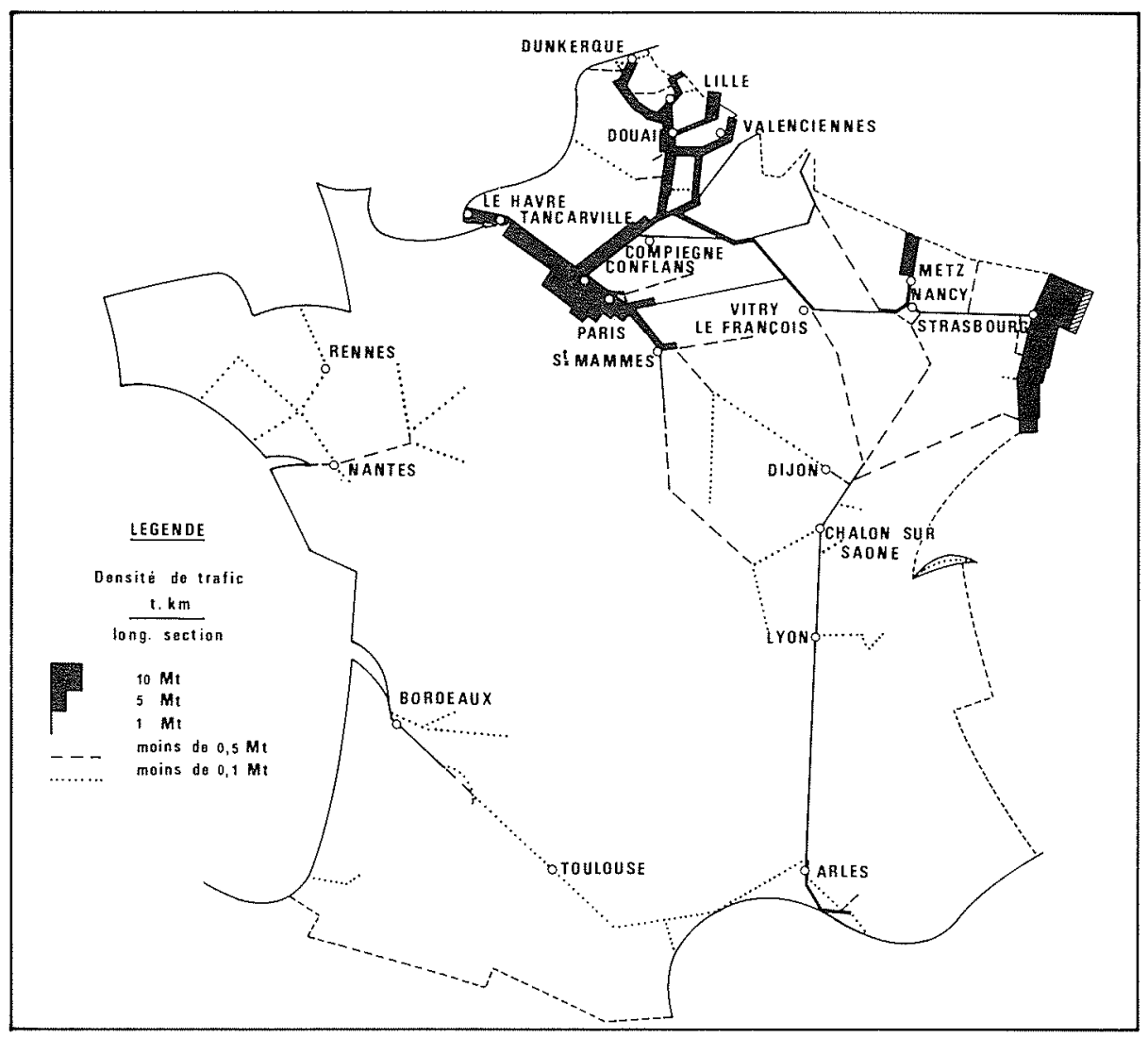

Figure 1

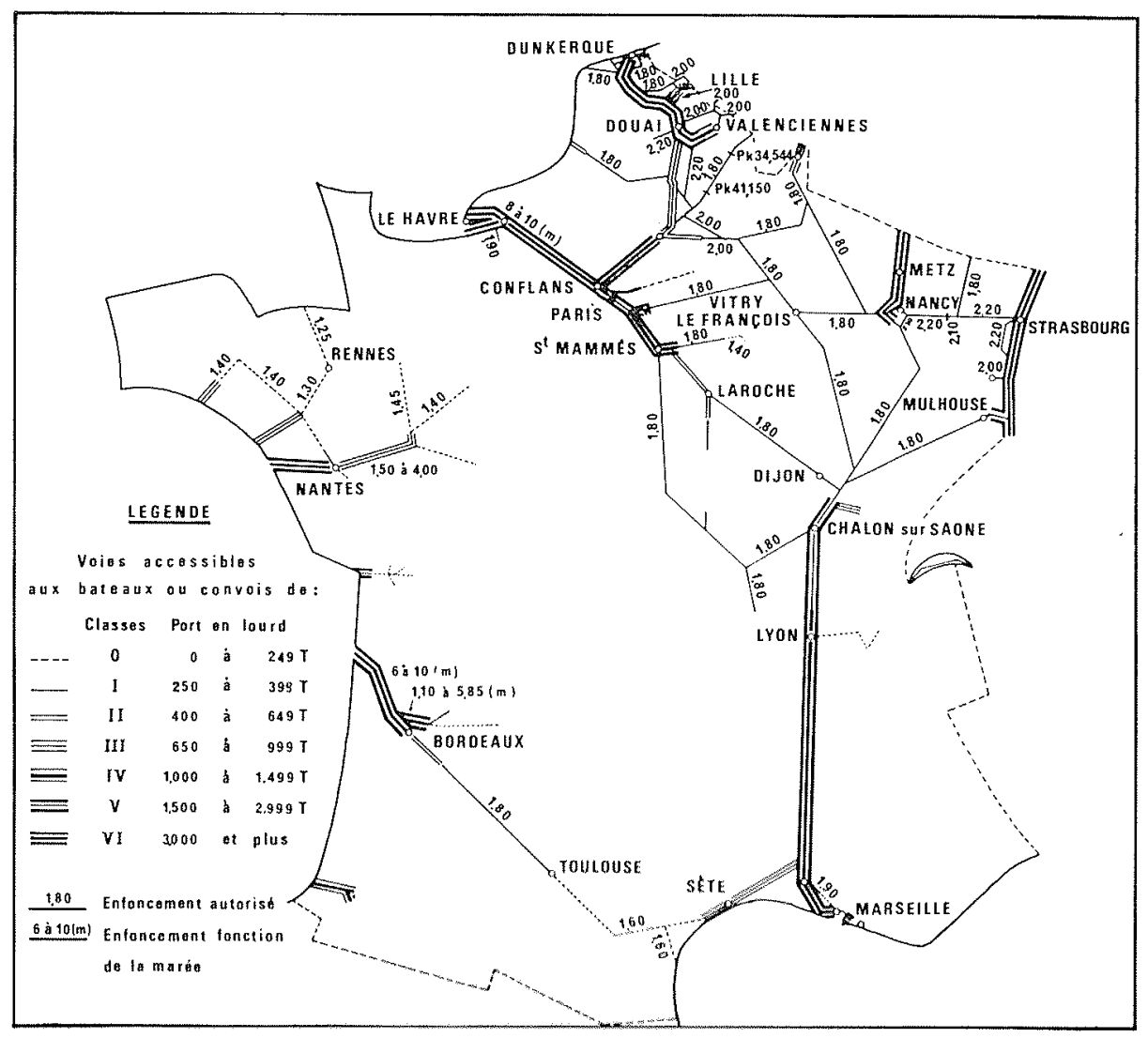

Finure 2 
Ensuite on améliora la navigation sur les rivières. Sur la Loire, le Rhône, le Rhin, on effectua des "aménagements à courant libre", c'est-à-dire de petites rectifications de tracé et des calibrages du chenal navigable pour augmenter les profondeurs, sans construction de barrages et d'écluses.

Cette technique n'était pas valable pour des rivières à débit d'étiage trop faible. Pour augmenter la hauteur d'eau disponible en tout temps on mit au point le procédé de la "canalisation" (des barrages relèvent le niveau de l'eau, la dénivellation aux barrages étant franchie par des écluses). Les premières canalisations ont été effectuées sur les rivières du bassin de la Seine (Seine, Yonne et Marne) et ont servi de modèles aux opérations analogues effectuées dans d'autres pays. Le mouillage minimal réalisé était le même que pour les canaux.

Le plan Freycinet (1880) fixa pour de longues années la consistance et les caractéristiques du réseau français. Le bateau-type était la péniche de $38,50 \mathrm{~m}$ de longueur et 5,05 $\mathrm{m}$ de largeur (ou "péniche flamande"), qui pouvait porter jusqu'à $350 t$ à l'enfoncement (ou tirant d'eau) de 2,20 m.

Sur les canaux, chaque péniche était halée, d'abord par des chevaux, puis par des tracteurs électriques sur rails à partir de 1895 , pour les canaux très fréquentés.

Sur les rivières, une ou plusieurs de ces péniches étaient tirées par un remorqueur. Pour accélérer l'éclusage de ces convois remorqués, il était intéressant de pouvoir placer dans le sas de l'écluse l'ensemble des bateaux et du remorqueur. Sur certaines voies importantes on construisit alors des écluses de plus grandes dimensions (Seine: $141 \times 17 \mathrm{~m}$, Oise : $125 \times 12 \mathrm{~m}$ ).

Avec la mise au point du moteur diésel, de plus en plus de bateaux furent des "automoteurs" pour lesquels on garda les mêmes dimensions que les péniches, sauf sur les grandes voies où on construisit des automoteurs plus grands (Kast campinois, chalands du Rhin).

Pour améliorer la liaison entre le bassin parisien et le Nord de la France, qui était assurée par le Canal de St-Quentin, on décida en 1903 de construire un nouveau canal, le "Canal du Nord", avec des écluses de $90 \times 6 \mathrm{~m}$ pouvant contenir 2 péniches Freycinet. Les travaux étaient réalisés aux 3/4 environ en 1914.

En 1913, le trafic sur les voies navigables françaises s'élevait à 42 millions de tonnes et 5,85 milliards de t.km.

Entre les deux guerres mondiales, on ne fit presque rien en France pour la navigation intérieure. Les travaux du Canal du Nord ne furent pas repris.

Aux Etats-Unis s'était développée sur les grands fleuves la technique nouvelle du poussage. Un convoi poussé est composé d'un certain nombre de "barges" qui sont des sortes de grandes caisses métalliques sans gouvernail et sans équipage. Ces barges sont réunies entre elles par des câbles et sont également attachées de la même façon devant un "pousseur", qui est l'équivalent du remorqueur. Quand il navigue, un convoi poussé constitue donc une unité monolithique. Cela procure des avantages importants par rapport au convoi remorqué : facilité de manœuvre, économie de personnel et d'énergie.

En 1938 , le trafic avait peu progressé en France (45 Mt).
Le parc fluvial français comprenait 12640 bateaux.

Les péniches flamandes (type Freycinet) représentaient $80 \%$ de ce parc. Seulement le quart était motorisé. Chacune de ces unités était exploitée par un marinier et sa famille, qui vivaient en permanence à bord. La plupart des bateaux appartenait aux mariniers. L'exploitation de la voie d'eau restait essentiellement artisanale.

$\mathrm{Au}$ lendemain de la guerre, la reconstruction s'est accompagnée d'une active motorisation du parc: en 1954, les automoteurs représentaient $40 \%$ environ de la capacité de transport. Aujourd'hui les bateaux tractés ont complètement disparu.

La navigation par convois poussés a commencé à se développer en Europe vers 1950, d'abord sur le Rhin.

Peu à peu, les dimensions des barges se sont unifiées. On considère maintenant comme un standard la barge de $76,50 \mathrm{~m}$ de longueur et $11,40 \mathrm{~m}$ de largeur, qui peut porter $2200 \mathrm{t}$ à l'enfoncement de $3,00 \mathrm{~m}$. Certaines barges peuvent aller jusqu'à $4 \mathrm{~m}$ d'enfoncement.

A partir de 1960, les voies françaises les plus importantes ont été modernisées pour permettre le passage de ces unités : Seine jusqu'à Bray, Moselle jusqu'à NeuvesMaisons, voie Dunkerque-Valenciennes avec antenne sur Lille, Oise jusqu'à Compiègne. Les aménagements du Rhin et du Rhône à l'aval de Lyon viennent d'être terminés. Dès l'origine les dimensions des écluses permettaient le passage de ces convois.

Le Canal du Nord a été enfin achevé en 1965. Il s'y est développé un type de navigation très performant au point de vue économique, sous la forme d'un convoi de 2 bateaux type Freycinet, un automoteur poussant une barge, qui est souvent un ancien automoteur ou une ancienne péniche tractée.

Dans tous les pays, du fait de l'évolution đes réseaux, les voies navigables présentent des caractéristiques, en particulier des dimensions d'écluses, assez hétérogènes. C'est ainsi qu'aux U.S.A., dont le réseau est le plus récent, il n'y a pas moins de 28 types de dimensions d'écluses.

Dans le cadre de la Communauté Economique Européenne, un effort a été fait pour classer et unifier les caractéristiques des réseaux de l'Europe de l'Ouest.

Les premières normes établies en 1954 par la Conférence Européenne des Ministres des Transports ont été rapidement dépassées par le développement du poussage. En 1962, 1'Union Internationale de Navigation Fluviale a préconisé des dimensions d'écluses qui sont pratiquement adoptées actuellement. La largeur est fixée à $12 \mathrm{~m}$. et la longueur à $110 \mathrm{~m}$ ou $185 \mathrm{~m}$ selon qu'on veut permettre le passage des convois d'une ou deux barges en flèche.

En France, la circulaire ministérielle du $1^{\text {er }}$ mars 1976 a fixé un classement des voies navigables et les dimensions correspondantes des écluses et de la section courante. Cette classification est homogène avec celle des autres pays de l'Europe de l'Ouest. Ci-contre sont données quelques indications.

\section{Le réseau français}

Il comprend actuellement (1979) $6534 \mathrm{~km}$ de voies fréquentées par la navigation commerciale. 
- $432 \mathrm{~km}(6,5 \%)$ ont des caractéristiques inféricures au gabarit Freycinet. Il s'agit des canaux les plus anciens qui n’ont jamais été améliorés (Canal du Midi, Canal du Nivernais, Voies de 1'Ouest). Le trafic y est faible (inférieur à $100000 \mathrm{t} / \mathrm{an}$ );

$-4355 \mathrm{~km}(66 \%)$ correspondent au gabarit Freycinet, ou à un gabarit légèrement supérieur (Classes I à III); Parmi ces voies, certaines n'ont plus qu'un trafic très faible, comme le Canal de Bourgogne. Il est envisagé de les concéder aux départements pour des utilisations à des fins touristiques.

Par contre, sur les voies qui correspondent à des itinéraires importants au plan économique, notamment pour la liaison avec les voies navigables de nos voisins, le trafic se maintient à plus de 1 million de $t$ /an.

C'est le cas pour les itinéraires allant du bassin de la Seine vers l'Est (Aisne, Canal de l'Aisne à la Marne, Canal latéral à la Marne, Canal de la Marne au Rhin) ou le Nord (Canal de St-Quentin et Canal du Nord). Sur ce dernier, le trafic est actuellement de l'ordre de 6 millions de $t / a n$, après avoir atteint plus de 8 millions de tonnes en 1970 .

- $1925 \mathrm{~km}$ de voies $(28 \%)$ sont à grand gabarit et permettent le passage de tous les grands automoteurs et des convois poussés composés d'au moins une barge de $76,50 \times 11,40 \mathrm{~m}$.

Cette catégorie comprend :

- Bray-Sur-Seine, avec des écluses de $185 \times 12 \mathrm{~m}$ à l'amont de Paris et à l'aval de $195 \times 18$ ou $24 \mathrm{~m}$;

- l'Oise à l'aval de Compiègne (écluses de $185 \times 12 \mathrm{~m}$ ) et le Canal lateral à l'Oise (écluses de $100 \times 12 \mathrm{~m}$ );

- le Rhône à l'aval de Lyon (écluses de $195 \times 12 \mathrm{~m}$ ) et la Saone à l'aval d'Auxonne (écluses de $185 \times 12 \mathrm{~m})$;

- La Moselle à laval de Neuves-Maisons (176 et $185 \times 12 \mathrm{~m})$

- Le Canal Dunkerque-Valenciennes avec son antenne sur Lille (ecluses de $144 \times 12 \mathrm{~m}$ ).

Notons que viennent de commencer les travaux de construction d'un canal Rhône-Fos destiné à relier à grand gabarit le port de Fos au Rhône, avec écluse de $185 \times 12 \mathrm{~m}$.

Le trafic sur les voies à grand gabarit représente $75 \%$ du trafic total en tkm. La voie la plus fréquentée est la Seine à l'aval de Paris (16 Mt/an) suivie de l'Oise canalisée $(5,8 \mathrm{Mt} / \mathrm{an})$ et de la Moselle (5,3 Mt/an) et du Canal Dunkerque-Valenciennes (4,0 à 4,9 Mt/an). Notons que le Rhin (Grand Canal d'Alsace) atteint $15 \mathrm{Mt} /$ an entre Strasbourg et Lauterbourg.

Le réseau français des voies navigables se caractérise par la coexistence de voies anciennes à petit gabarit et de quelques axes à grand gabarit qui ne représentent guère plus du quart de la longueur totale mais qui supportent les 3/4 du trafic. Il faut noter cependant qu'une part assez importante de ce trafic est assurée par des bateaux à petit gabarit.

Il y a deux raisons à cela. D'une part, le tonnage unitaire des expéditions ne justifie pas toujours le recours au grand gabarit. D'autre part, les itinéraires comprennent souvent des voies à petit gabarit.

L'idée de relier entre eux ces axes par des voies à grand gabarit est bien naturelle et des avant-projets ont çtế commencés dès 1965. L'opération la plus importante, la liaison Saône-Rhin, a été déclarée d'utilité publique en 1978.

Les études se poursuivent actuellement sur la liaison Seine-Nord qui devrait relier l'Oise canalisée, d'où le bassin de la Seine, au Canal Dunkerque-Valenciennes.

\section{Les voies navigables de l'Europe de l'Ouest}

Nous ne traiterons que des réseaux belges, néerlandais et allemands. Dans ces pays, l'évolution a été analogue à celle constatée en France. Cependant, la modernisation des voies s'est effectuée plus tôt et la proportion des voies à grand gabarit est plus élevée.

Le réseau belge est constitué essentiellement par un ensemble de voies convergeant vers le port d'Anvers (Lys, Escaut, Dender, Canal Bruxelles-Charleroi, Canal Albert) qui sont reliées à la périphérie par la Meuse, le Canal du Centre et le Canal Nimy-Blaton. Les communications sont nombreuses avec le réseau français, par la Lys, l'Escaut, le Canal de Roubaix, la Sambre et la Meuse, et avec le réseau hollandais, surtout par la Meuse et par l'Escaut. Ces voies ont des caractéristiques assez hétérogènes. D'autre part, le Canal Gand-Terneuzen relie Gand directement à l'embouchure de l'Escaut. Il est de Classe VI.

Les voies les plus fréquentées sont l'Escaut ( $8 \mathrm{Mt} / \mathrm{an}$ ) et surtout l'axe contitué par la Meuse et le Canal Albert (20 Mt/an).

La modernisation du réseau belge a été définie par la loi du 6 mars 1957, l'objectif étant le passage de l'automoteur dit "européen" de 1350 t. Les travaux d'aménagement' se poursuivent notamment sur le Canal du Centre où va être construit près de Strepy un double ascenseur à contrepoids avec un bac de $100 \times 12,50 \mathrm{~m}$, rachetant une chute de $73 \mathrm{~m}$ de hauteur.

Sur la Meuse et le Canal Albert il est prévu un aménagement pour permettre le passage de convois de 9000 t constitués par 4 grandes barges.

En vertu d'un traité signé avec la Hollande en 1967 il vient d'être aménagé une liaison Escaut-Rhin per. mettant un itinéraire direct entre Anvers et l'embou. chure du Rhin, et Rotterdam, avec la construction des écluses de Kreekrak (Pays-Bas) de $350 \times 24$ m qui permettront le passage des plus grands convois et de cer. taines unités maritimes.

Il est.à noter que cette voie aboutit dans le lac de Zélande dont l'eau est presque douce en raison de l'apport de 1'Escaut, tandis que le bief vers le Sud communique avec le Port d'Anvers, donc avec la mer. Aussi, il est prévu à ces écluses un dispositif anti-sel pour éviter d'augmenter la salure du Lac de Zélande.

Le réseau hollandais est le plus ancien et le plus dense. Comme en France, la plupart des voies étaient tracées au milieu du siècle dernier et il subsiste encore une proportion importante (près du quart) de voies non accessibles aux bateaux de $350 \mathrm{t}$.

Dans la moitié Sud du pays, le réseau converge vers Rotterdam qui, signalons-le, est le plus grand port du monde, son trafic a atteint $350 \mathrm{Mt} / \mathrm{an}$.

L'axe principal est représenté par les deux grandes voies à peu près parallèles qui sont la Meuse et le Rhin. 


\begin{tabular}{|c|c|c|c|c|c|c|}
\hline \multirow[b]{2}{*}{ Classes } & \multicolumn{2}{|l|}{ Unité-type } & \multirow{2}{*}{$\begin{array}{l}\text { Dimensions } \\
\text { des } \\
\text { écluses m }\end{array}$} & \multicolumn{3}{|c|}{ Section courante } \\
\hline & $\begin{array}{c}\text { Dimensions } \\
\mathrm{m}\end{array}$ & $\begin{array}{c}\text { Port en lourd } \\
t\end{array}$ & & $\begin{array}{l}\text { Largeur au } \\
\text { plafond } m\end{array}$ & $\begin{array}{c}\text { Mouillage } \\
\mathrm{m}\end{array}$ & $\begin{array}{c}\text { Section } \\
\text { mouillée } m^{2}\end{array}$ \\
\hline Petit gabarit & & & & & & \\
\hline 1 & $\begin{array}{c}38,50 \times 5,05 \\
\text { (Bateau Freycinet) }\end{array}$ & 350 à 400 & $40 \times 6$ & 15 & 3,00 à 3,50 & 62 à 72 \\
\hline $\begin{array}{l}11 \\
111\end{array}$ & $\begin{array}{l}\text { pour memolre } \\
90 \times 5,05\end{array}$ & 700 à 775 & $92 \times 6$ & 18 & 3,00 à 3,50 & 72 à 82 \\
\hline Grandgabarit & & & & & & \\
\hline IV & $\begin{array}{c}105 \times 11,40 \\
\text { (convoi d'une grande barge) }\end{array}$ & 1800 à 2200 & $110 \times 12$ & 34 à 36 & 4,00 à 4,50 & 170 à 200 \\
\hline V & $\begin{array}{c}180 \times 11,40 \\
\text { (convoi de } 2 \text { grandes barges } \\
\text { enfoncement } 2,50 \mathrm{~m} \text { ) }\end{array}$ & 3600 & $185 \times 12$ & 34 & 4,00 & 170 \\
\hline VI & $\begin{array}{c}180 \times 11,40 \\
\text { (convoi de } 2 \text { grandes barges } \\
\text { enfoncement } 3,00 \mathrm{~m} \text { ) }\end{array}$ & 4400 & $185 \times 12$ & 36 & 4,50 & 200 \\
\hline
\end{tabular}

Tableau comparatif des longueurs des réseaux et des trafics totaux (en 1979 pour la France, 1978 pour les autres pays)

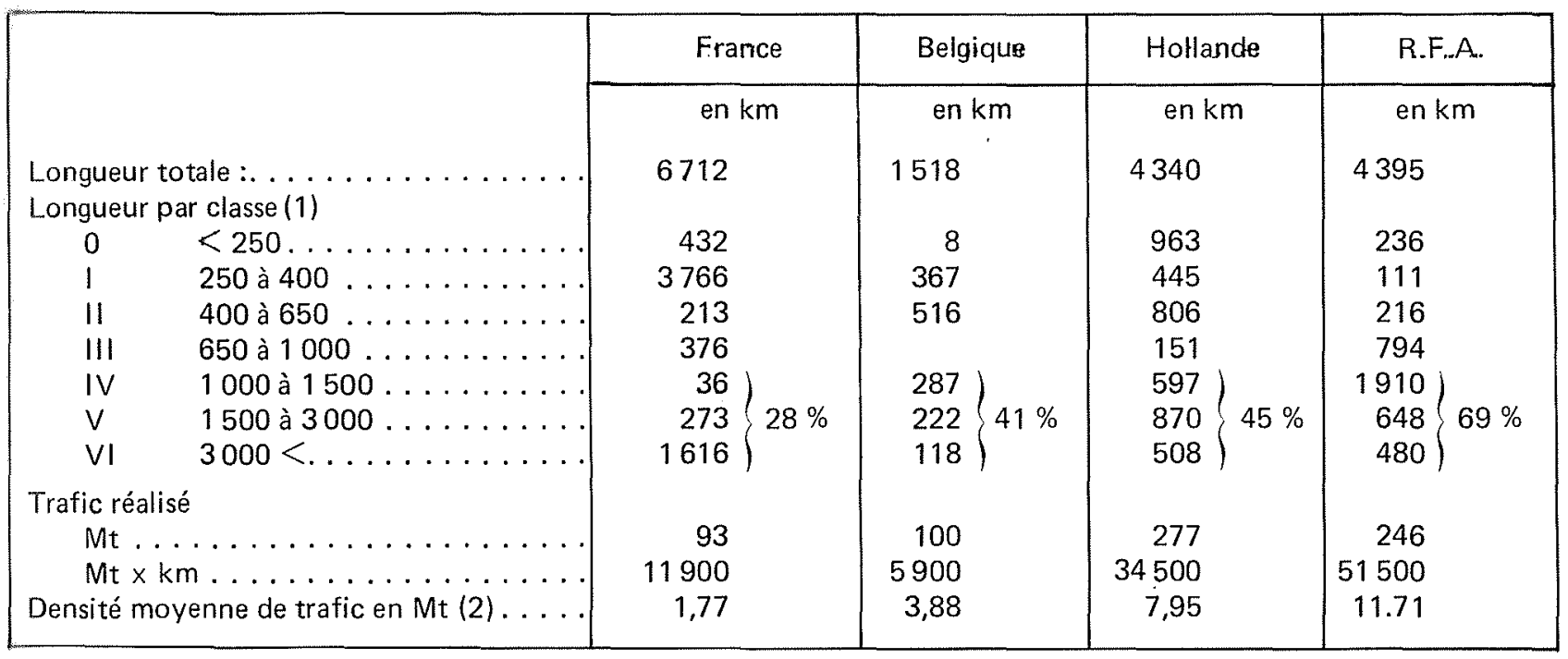

(1) Classes adoptées en France et limites de port en lourd pour les autres pays.

(2) Trafic total en tkm divisé par la longueur totale du réseau.

Sur ce dernier les écluses ont $185 \times 22,80 \mathrm{~m}$ et permettent donc le passage du convoi de 4 barges ou de 2 convois de 2 barges.La Meuse est équipée avec des écluses de $100 \times 12 \mathrm{~m}$.

La liaison entre Rotterdam et Amsterdam est assurée par 3 itinéraires, le plus important (AmsterdamRijnkanaal) comporte des écluses de $350 \times 13 \mathrm{~m}$.

Le Canal Escaut-Rhin et le Canal Gand-Terneuzen assurent un itinéraire direct vers le réseau belge et le Nord de la France.

Dans la zone des polders au Nord d'Amsterdam s'étend un réseau très ramifié de canaux pour bateaux de 600 à $1350 \mathrm{t}$.

En République Fédérale Allemande, l'artère principale est certainement le Rhin qui dessert les grandes zones industrielles. Sa longueur est de $480 \mathrm{~km}$ entre les frontières française et hollandaise. Il n'y a aucune écluse, le Rhin étant aménagé à courant libre dans cette zone. De ce fait, les profondeurs disponibles sont fonction du débit. L'objectif est d'obtenir une profondeur minimale de 2,10 m. Des travaux importants viennent d'être effectués au passage du "Bingerlock" entre Karlsruhe et St-Goar, où le Rhin présente des pentes plus accentuées à la traversée du plateau schisteux rhénan.

Des convois de 4 grandes barges peuvent normalement circuler sur le Rhin. Le trafic à la frontière hollandaise dépasse $200 \mathrm{Mt} / \mathrm{an}$, ce qui représente $80 \% \mathrm{du}$ trafic total de la R.F.A.

La Moselle a un statut international car elle a été canalisée en vertu d'un traité avec la France et le Luxembourg. Avec ses écluses de $176 \times 12 \mathrm{~m}$ elle permet le passage de convois de $3000 \mathrm{t}$ pour la liaison avec le bassin lorrain. 
La Sarre vient également d'être canalisée par la R.F.A.

Le Main et le Neckar complètent les antennes du Rhin.

Le réseau de la R.F.A. comprend, d'autre part, des fleuves comme l'Ems, la Weser, l'Elbe, aménagés pour le passage de convois de 2000 à $3000 \mathrm{t}$, qui sont reliés par le Mittelland-Kanal de caractéristiques plus réduites (1350 t).

Il vient d'être construit un Canal latéral à l'Elbe pour relier ce fleuve au Mittelland-Kanal sans passer par la R.D.A. Un double ascenseur à contrepoids de $38 \mathrm{~m}$ de hauteur a été réalisé pour franchir la chute d'Uelzen.
Rappelons enfin que la liaison Rhin-Main-Danube sera bientôt achevée. Avec des écluses de $185 \times 12 \mathrm{~m}$, elle permettra le passage des grands automoteurs et de convois de 2 grands barges.

D'une façon générale, on voit que ces pays possèdent un réseau ayant une proportion de voies à grand gabarit plus grande qu'en France. Par contre, il y a en France plus de voies de classe VI, à la fois en valeur absolue et en pourcentage.

Cela doit provenir du fait que la modernisation $d u$ réseau a commencé plus tard en France, au moment où la navigation par convois poussés de 2 grandes barges s'avérait la solution d'avenir pour les voies très fréquentées. 